\title{
Thermally Excited Resonating Membrane Mass Flow Sensor*
}

\author{
SIEBE BOUWSTRA, PAUL KEMNA and ROB LEGTENBERG \\ Department of Applied Physics, Research Unit Sensors and Actuators, University of Twente, P.O. Box 217, \\ 7500 AE Enschede (The Netherlands)
}

(Received December 15, 1988; in revised form March 17, 1989; accepted April 21, 1989)

\begin{abstract}
A mass flow sensor based on the frequency shift of a resonating microstructure is being developed, using a measurement principle of the thermoanemometry type. The sensor is to be applied for mass flows up to 10 standard cubic centimeters per minute $\left(\mathrm{sccm} ; 10 \mathrm{sccm}=0.17 \mathrm{mg} \mathrm{s}^{-1}\right)$, with a high sensitivity, a high resolution and a fast response. Here we report on the first prototype consisting of a $2 \mu \mathrm{m}$ thick membrane: the temperature elevation of the thermally excited vibrating membrane affects its resonance frequency. The three-dimensional heat transfer within the membrane and the mass flow is modeled, and expressions are derived for the resonance frequencies of initially curved and stressed membranes. Experiments have been carried out for nitrogen flows of up to $500 \mathrm{sccm}$ passing over thermally excited membranes. Predicted and measured values for the shift of the resonance frequency agree well. The sensitivity of the average temperature elevation to the mass flow is quite small: at $10 \mathrm{sccm}$ the cooling effect of the mass flow is only $0.2 \%$ of the heat loss by conduction to the substrate. At a resonance frequency of $5.0 \mathrm{kHz}$, and an average temperature elevation of the membrane of $8{ }^{\circ} \mathrm{C}$, this still leads to a frequency change of $13 \mathrm{~Hz}$ in the mass flow range from zero to $10 \mathrm{sccm}$. Suggestions are presented for increasing the sensitivity of the sensor.
\end{abstract}

\section{Introduction}

Currently, there is a growing demand for highperformance mass flow sensors, notably in the IC industry [1]. Mass flow measurement based on dynamic pressure or on friction losses would require a very sensitive differential pressure sensor. Also, these measurements are indirect: an additional measurement would be required to determine the actual mass flow. Direct measurement of mass

\footnotetext{
*Paper presented at EUROSENSORS 87, First European Conference on Sensors and their Applications, Cambridge, U.K., September 22-24, 1987.
}

flow could be obtained via Coriolis or gyroscopic forces. This would require an even more sensitive sensor, as well as a complicated mechanical structure.

The best option to measure the mass flow of a gas is to make use of the ability of the gas to transport heat by forced convection [2]. The specific heat of gases, usually of the order of magnitude of $10^{3} \mathrm{~J}\left(\mathrm{~kg}^{\circ} \mathrm{C}\right)^{-1}$, is virtually independent of ambient conditions [3]. In this type of flow meter a heater is in contact with the gas, and the generated heat is partly transported away by convection. Either the resulting temperature elevation of the heater itself is measured (the thermo-anemometer type, Fig. 1(a)), or the temperature difference between two points within the gas is measured, one point upstream and one downstream of the heater (the calorimetric flowmeter, Fig. 1(b)). These configurations are also used for velocity measurements.

The actual measurement of the mass flow is usually done in a shunt arrangement, Fig. 1(c). With an adjustable restriction in the by-pass, the ratio of the flow through the sensor channel to the total mass flow can be pre-set, providing both flows are of the same regime, i.e., either laminar or turbulent. In the conventional thermal mass flow sensor, the sensor channel consists of a hollow pipe with a resistive heater and resistive temperature sensors wound around the pipe. As the heated zone has an extensive length in relation to the considered gas flows, the temperature profile within the gas can be regarded as being fully developed, i.e., a flat profile across each cross-section. The contribution to the total heat transfer by thermal conduction within the gas is then negligible. However, the heat loss by thermal conduction within the pipe wall leads to a poor sensitivity, and as the relatively large heat capacity of the pipe is located between the heating element and the fluid, a slow response results. For a high-performance measurement, it is preferable to have the sensor within the channel.

IC technology provides the opportunity to realize flow sensors with miniature heating elements and temperature sensors [4]. Micromachining of silicon provides the opportunity for compatible realization of flow channels. The sensing area of 

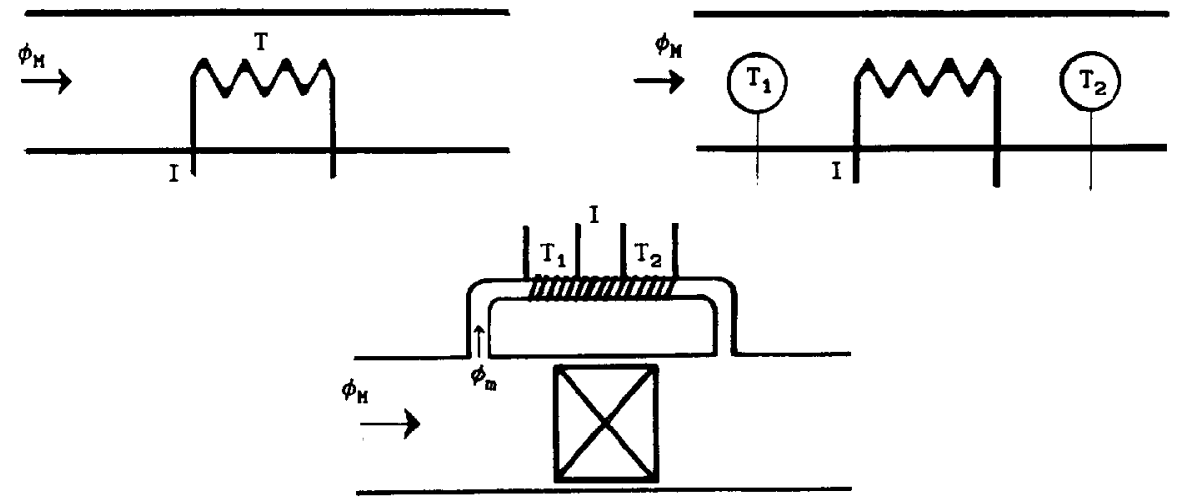

Fig. 1. Conventional mass flow measurement: (a) thermo-anemometer; (b) calorimetric flowmeter; (c) shunt arrangement.

the sensor can be suspended within the flow channel, by preferential etching of the substrate [5-9]. As the coefficient of heat transfer by thermal conduction to the substrate (in $\mathrm{W}^{\circ} \mathrm{C}^{-1}$ ) is small for these sensors, their temperature elevation is very sensitive to changes in the mass flow. Also, their relatively even smaller heat capacities (in $\mathbf{J}^{\circ} \mathrm{C}^{-1}$ ) lead to fast response times (heat capacity divided by coefficient of heat transfer) of only a few milliseconds or less.

We are investigating the possibilities of a resonating micromechanical structure for mass flow measurement within a microchannel. The principle under consideration is that of the thermoanemometer type. Besides the general advantages of resonant sensors, like stability and a semi-digital output [10], resonating membrane sensors have a high sensitivity to planar stress. With the latter caused by thermal expansion, this sensor principle has the potential of a mass flow measurement with an unprecedentedly high resolution. Our aim is to develop a mass flow sensor for small mass flows up to $10 \mathrm{sccm}\left(0.17 \mathrm{mg} \mathrm{s}^{-1}\right)$, operating at a temperature elevation of less than $20^{\circ} \mathrm{C}$, with high demands for accuracy, resolution, repeatability and response time. In this paper we report on our first prototype, the resonating membrane mass flow sensor.

First we describe the operation and the fabrication of the sensor, as well as the experimental set-up. Next we describe two models for the heat transfer from the membrane to the gas, i.e., a very simple model for a first estimation, and a numerical model for a more accurate prediction. After presenting a model for the resonance frequency of a stretched and curved membrane, we present the results of these models and compare these with the experimental results. Suggestions are made for the improvement of the sensitivity of the sensor.

\section{Operation}

The operation of the resonating membrane mass flow sensor is illustrated in Fig. 2. By applying a d.c. voltage across the centre resistor, a distributed static heat is generated. A fluid passes over the heated membrane. The heat is transferred to the fluid and also, at the edges, to the substrate. The resulting average temperature elevation of the membrane is dependent on the cooling effect of the passing fluid.

For the forced vibration of the membrane, we make use of thermal excitation and piezoresistive detection. For the excitation, an a.c. voltage is superimposed on the d.c. voltage applied to the centre resistor described above. The dynamic component of the generated heat leads to a periodic thermal expansion of the resistor, thereby inducing a bending mode vibration of the membrane. Near the clamped edge of the membrane four piezoresistors are arranged in a Wheatstone bridge to detect the vibration of the membrane.

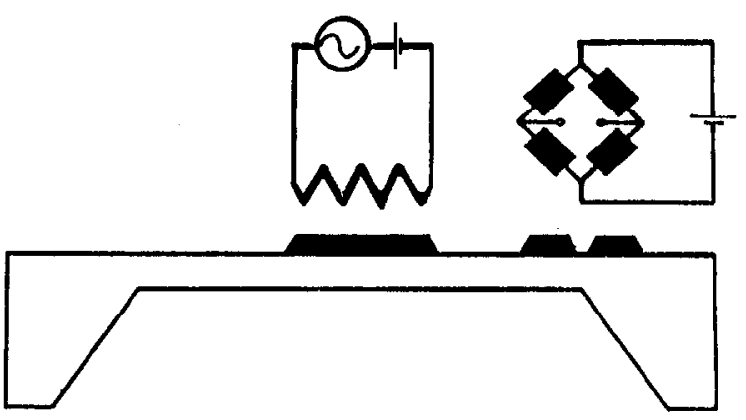

Fig. 2. Principle of operation of resonating membrane mass flow sensor: centre resistor for thermal excitation of the bending mode vibration; piezoresistors adjacent to the edge of the membrane for detection of the vibration. 
With d.c. voltages applied to the centre resistor and to the Wheatstone bridge, and the a.c. output voltage of the latter amplified and superimposed on the d.c. voltage applied to the centre resistor, we have an electro-thermo-mechanical oscillator [11].

The resonance frequency of the membrane depends on the flexural rigidity and on planar stresses. The temperature elevation of the membrane relative to the support causes a compressive planar thermal stress, thereby decreasing the resonance frequency. Cooling of the membrane by the mass flow causes an increase of the frequency. This frequency change is now a measure for the mass flow passing the membrane.

\section{Fabrication}

A $2^{\prime \prime}$ n-type (100)-silicon wafer with a resistivity of $1-10 \Omega \mathrm{cm}$ and a thickness of $285 \pm 15 \mu \mathrm{m}$ is used for the fabrication of the sensor. Conventional techniques are used for thin-film deposition, doping, lithography and etching.

First, a $1 \mu \mathrm{m}$ thick oxide film is grown in a damp atmosphere at $1150^{\circ} \mathrm{C}$. After the oxide film has been patterned with etch-windows on the back side of the wafer, and has been stripped off the polished side, a $\mathrm{p}^{++}$-layer is formed on the polished side by drive-in diffusion from a CVD boron glass thin film. The high boron concentration provides an etch-stop at a later stage in the process sequence. The glass film later serves as an electrically insulating layer.

Next, a $400 \mathrm{~nm}$ in situ phosphorus-doped polysilicon film is deposited by LPCVD (at $675^{\circ} \mathrm{C}$

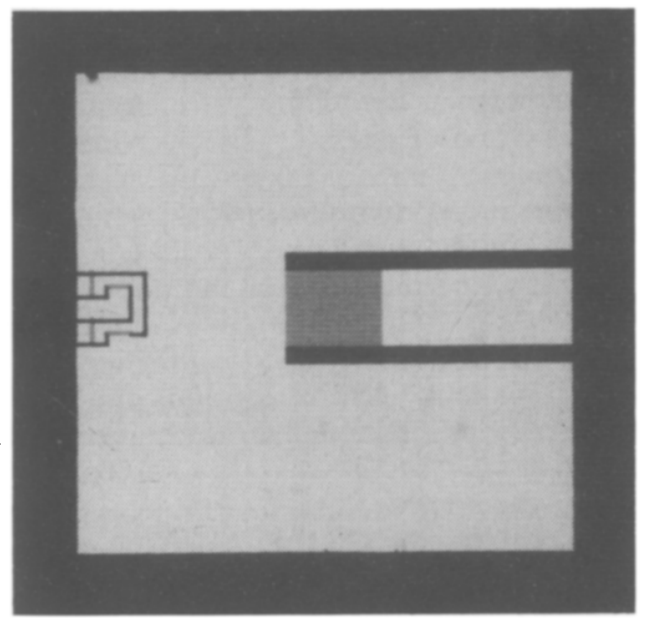

Fig. 3. Photograph of $2 \times 2 \mathrm{~mm} \times 2 \mu \mathrm{m} \mathrm{p}^{++}$-silicon membrane, with polysilicon resistors for excitation (centre) and detection (edge) of the vibration, and with aluminium connection wires (illumination from both sides). and at $0.5 \mathrm{mbar}$, with a silane-to-phosphine mass flow mixing ratio of $1: 800$ ), with a sheet resistivity of $130 \Omega_{\square}$ after annealing at $900{ }^{\circ} \mathrm{C}$ for $30 \mathrm{~min}$ utes. After definition of the polysilicon resistors by wet chemical etching, $400 \mathrm{~nm}$ aluminium is deposited by electron beam evaporation and patterned for interconnection. Finally, the wafer is etched from the back side in a KOH/IPA solution at $70^{\circ} \mathrm{C}$. Etching stops automatically at the region with a sufficiently high boron concentration [12], yielding membranes with a thickness equal to approximately $60 \%$ of the junction depth.

This fabrication technique yields thin $\mathrm{p}^{++}$ silicon membranes with the thin film patterns on top. Figure 3 shows a photograph of a square membrane with lateral dimensions of $2 \mathrm{~mm}$, and a thickness of $2 \mu \mathrm{m}$.

\section{Experimental Set-up}

Figure 4 shows the experimental set-up for testing the sensor. A controlled nitrogen gas flow is passed over the heated membrane through a flow channel with $4 \times 4 \mathrm{~mm}^{2}$ cross-section. For the vibration of the membrane, the a.c. output voltage of a gain/phase analyser is amplified, and superimposed on the output voltage of a d.c. source. This is then applied over the excitation resistor at the centre of the membrane. A second d.c. voltage is applied across the Wheatstone

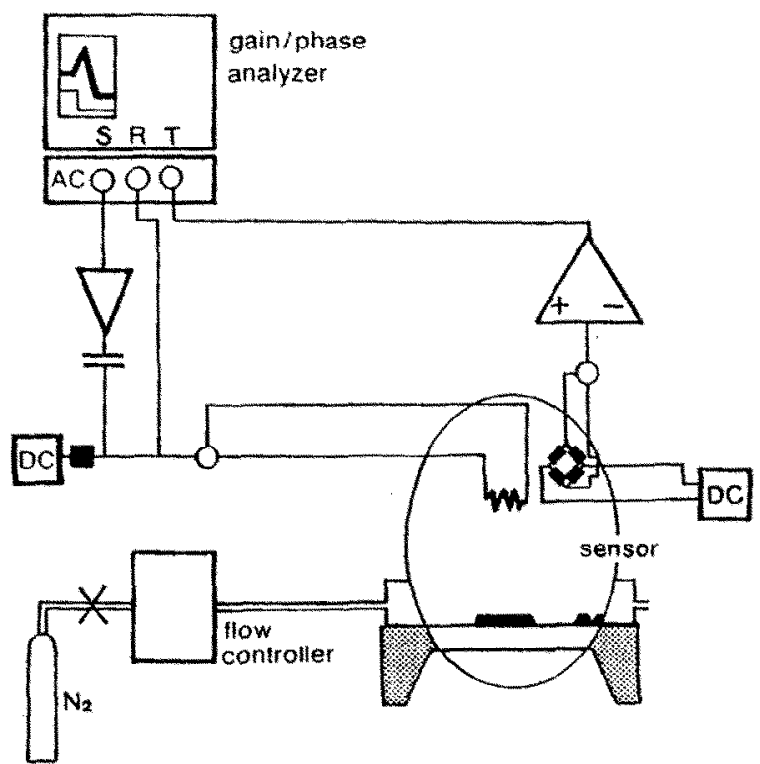

Fig. 4. Experimental set-up: nitrogen gas flow through channel over heated membrane. The frequency response of the vibrating membrane with thermal excitation and piezoresistive detection is determined using a gain/phase analyser. 
bridge. Via a differential amplifier, the bridge output is then connected to the analyser. By sweeping the excitation frequency, the analyser measures the transfer of this two-port electrothermo-mechanical device. From this, the resonance frequency is determined, which is now a function of the static heat generated on the membrane, and of the cooling effect of the nitrogen flow.

\section{Heat Transfer}

The heat generated on the membrane is transferred by conduction within the membrane to its cold edges, and by conduction and forced convection within the gas. Calculations have shown that contributions by free convection and by radiation are negligible. First, a very simple model is derived to obtain a rough idea of the effect of the mass flow on the temperature elevation of the membrane. For a more accurate analysis, a more complex model is constructed, which is solved using the finite-difference method.

\section{Simple Model}

The simple model describes the heat transfer with two parallel lumped elements: the membrane and the gas. The heat transfer within the membrane is described by the two-dimensional Laplace equation. Solving this equation for a homogeneous circular membrane with coefficient of heat conduction $\lambda\left(\mathrm{W}\left(\mathrm{m}^{\circ} \mathrm{C}\right)^{-1}\right)$ and uniform thickness $t(\mathrm{~m})$, with a generation of heat per unit time $H(\mathrm{~W})$ concentrated at its centre and with cold edges, the average temperature elevation $\Delta T_{\mathrm{av}}\left({ }^{\circ} \mathrm{C}\right)$ is found from: $\Delta T_{\mathrm{av}}=H /(4 \pi \lambda t)$, independent of the diameter, and half this value for a heat source uniformly distributed over the entire membrane.

Assuming that the gas does not affect the temperature profile of the membrane, and assuming (for zero temperature elevation of the channel walls) that the gas reaches an average temperature elevation that is half the average temperature elevation of the membrane, then the heat transfer by convection will be $c_{p} \phi_{\mathrm{m}} \Delta T_{\mathrm{av}} / 2$, with $c_{p}$ the specific heat of the gas $\left(\mathrm{J}\left(\mathrm{kg}^{\circ} \mathrm{C}\right)^{-1}\right)$, and $\phi_{\mathrm{m}}$ the mass flow $\left(\mathrm{kg} \mathrm{s}^{-1}\right)$. Omitting the heat conduction to the channel walls, the total heat transfer (in the case of the concentrated heat source) is then:

$$
\begin{aligned}
& H=\left(\chi_{\lambda}+\chi_{\phi}\right) \Delta T_{\mathrm{av}} \\
& \chi_{\lambda}=4 \pi \lambda t \\
& \chi_{\phi}=\frac{1}{2} c_{p} \phi_{m}
\end{aligned}
$$

where $\chi_{\lambda}$ and $\chi_{\phi}$ are the coefficients of heat transfer $\left(W^{\circ} C^{-1}\right)$ of the membrane and of the gas respectively, both expressed in terms of the average temperature elevation of the membrane.

With the maximum average temperature elevation $\left(\Delta T_{\mathrm{av}}\right)_{\max }$ occurring for $\phi_{m}=0$, eqn. (1) can be rewritten:

$$
\begin{aligned}
& \theta=(1+\xi)^{-1} \\
& \xi=\chi_{\phi} / \chi_{\lambda} \\
& \theta=\Delta T_{\mathrm{av}} /\left(\Delta T_{\mathrm{av}}\right)_{\max } \\
& \left(\Delta T_{\mathrm{av}}\right)_{\max }=H / \chi_{\lambda}
\end{aligned}
$$

with $\chi_{\phi}$ and $\chi_{\lambda}$ as in eqn. (1), and where $\theta$ is the dimensionless temperature elevation and $\xi$ the dimensionless coefficient of heat transfer by convection. Equations (2) will also hold for the results of the complex model, as well as for the experimental results, if $\chi_{i}$ is defined as the resulting total coefficient of heat transfer for $\phi_{\mathrm{m}}=0$, and $\xi$ as the resulting normalized increase of the total coefficient of heat transfer with $\phi_{\mathrm{m}}$.

From eqn. (2) we see that for a given maximum allowable average temperature elevation $\left(\Delta T_{\mathrm{av}}\right)_{\max }$, and with the flow sensor operating at a constant heat generation $H$, the differential sensitivity of the average temperature to a change in the mass flow, $\partial T_{\mathrm{av}} / \partial \phi_{\mathrm{m}}$, decreases with increasing $\xi$. The lowest value of this sensitivity, which occurs at the maximum of the mass flow range, will be optimal if the maximum value of $\xi$ equals one (provided $\xi$ is proportional to the mass flow).

\section{Complex model}

For a more accurate analysis of the heat transfer, we have to take into account the unevenly distributed character of the temperature elevation of the membrane and of the gas. The mechanisms for the heat transfer that are taken into account are illustrated in Fig. 5: two-dimensional conduction of heat within the membrane, forced convection by the gas in the direction of the flow, and conduction of heat within the gas in planes perpendicular to the flow direction. Conduction of heat within the gas in the flow direction is omitted for practical reasons. The temperature profiles of the membrane and of the gas are coupled: at their interface the temperature elevation of the gas equals that of the membrane. Also, the heat flux into the gas is an additional distributed heat sink for the membrane. The resulting partial differential equations for the distributed temperature elevations of both the membrane and the gas, together with their boundary conditions, are then: 


$$
\begin{aligned}
& \lambda t\left\{\partial^{2} T_{\mathrm{m}} / \partial x^{2}+\partial^{2} T_{\mathrm{m}} / \partial y^{2}\right\}=-\phi(x, y) \\
& \rho c_{p} u(y, z) \partial T_{\mathrm{g}} / \partial x=\lambda_{\mathrm{g}}\left\{\partial^{2} T_{\mathrm{g}} / \partial y^{2}+\partial^{2} T_{\mathrm{g}} / \partial z^{2}\right\} \\
& \left.T_{\mathrm{g}}\right|_{z}=0=T_{\mathrm{m}} \\
& \phi(x, y)=\phi_{0}(x, y)-\phi_{\mathrm{g}}(x, y) \\
& \phi_{\mathrm{g}}(x, y)=-\lambda_{\mathrm{g}} \partial T_{\mathrm{g}} /\left.\partial z\right|_{z=0} \\
& T_{\mathrm{m}}=0 \text { for } x=0, x, y=0, y=l \\
& T_{\mathrm{g}}=0 \text { for } x=0, y=l, z=h
\end{aligned}
$$

where $T_{\mathrm{m}}=T_{\mathrm{m}}(x, y)$ and $T_{\mathrm{g}}=T_{\mathrm{g}}(x, y, z)$ are the temperature elevations of the membrane and of the gas respectively, $x, y$ and $z$ are the Cartesian coordinates as shown in Fig. 5, with $z=0$ at the interface between membrane and gas, $\rho, u(y, z)$ and $\lambda_{\mathrm{g}}$ are the density, the velocity profile and the coefficient of heat conduction of the gas respectively, and $\phi_{0}(x, y)$ and $\phi_{\mathrm{g}}(x, y)$ the distributed heat generation on the membrane and the heat flux from the membrane to the gas respectively; $l$ is both the lateral dimension of the square membrane and the width of the flow channel, $h$ is the height of the channel. In our calculations we assumed a velocity profile $u(y, z)$ parabolically dependent on $z$, and independent of $y$. The dependences of the material properties on temperature and pressure are neglected. The reason why we account for the conduction of heat within the gas in the $y$-direction will be clarified in the Discussion.

Equations (3) are solved using the finite-difference method, see Appendix. Solving the complete problem directly would be very complex. Instead, an iteration procedure is followed. First, the temperature elevation of the membrane is calculated, ignoring the gas. Next, this temperature elevation is taken as a boundary condition for the calculation of the temperature profile in the gas. The region above the membrane is divided into a finite number of planes perpendicular to the flow direction. As the conduction of heat in the flow direction is omitted, information on the temperature profile does not flow upstream. With a zero temperature elevation for the gas entering the area above the membrane, the temperature profile of the gas can therefore be determined solving the two-dimensional equations for the subsequent planes.

From the temperature profile of the gas, or more specifically, from the gradient perpendicular to the interface with the membrane, the heat flow through the interface into the gas is determined. This is accounted for as an additional heat sink in solving the temperature elevation of the membrane in the next iteration step. After three to five iteration steps, the solution has converged.

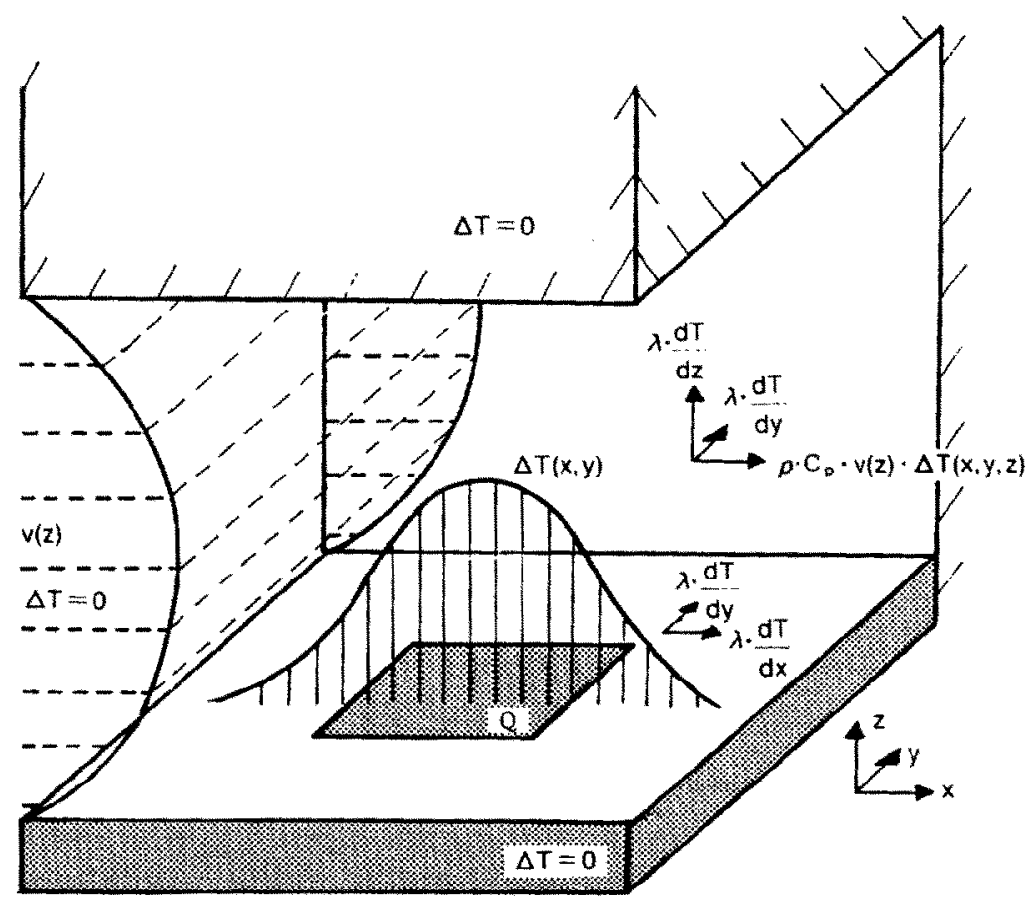

Fig. 5. Components of heat transfer, boundary conditions and gas velocity profile taken into account in the complex model: two-dimensional conduction within the membrane, forced convection by the gas flow, and two-dimensional conduction within the gas in planes perpendicular to the flow direction; cold walls and cold incoming gas, parabolic velocity profile. 


\section{Results}

According to the simple model (eqns. (1)), the coefficient of heat transfer of a square membrane with thickness $t=2 \mu \mathrm{m}$ and $\lambda=157$ $\left(\mathrm{W}\left(\mathrm{m}^{\circ} \mathrm{C}\right)^{-1}\right)$ is $3.9 \times 10^{-3} \mathrm{~W}^{\circ} \mathrm{C}^{-1}$. The coefficient of heat transfer by convection, for a mass flow of $10 \mathrm{sccm}$, of a gas with $c_{p}=$ $1000 \mathrm{~J}\left(\mathrm{~kg}^{\circ} \mathrm{C}\right)^{-1}$, is $0.08 \times 10^{-3} \mathrm{~W}^{\circ} \mathrm{C}^{-1}$. The relative contribution of the latter is then $2 \%$. Applied values for material properties were taken from [13] for single-crystal silicon for the membrane and for nitrogen for the gas, both at room temperature.

For more accurate data we have to look at the results of the complex model. A typical result of the calculation is illustrated in Fig. 6, where dissipation in the piezoresistors was neglected. Figure 6(a) is a plot of contour lines of the temperature elevation of the membrane, with a three-dimensional plot inserted. Despite the cooling effect of the mass flow in the $x$-direction, this profile is still quite symmetric, and is virtually the same as that for a circular membrane. It appears that the simple model is adequate for the heat transfer by conduction within the membrane.

Figure $6(b)$, however, illustrates the influence of the mass flow in a more pronounced way. It shows contour lines for the heat flux from the membrane to the gas, again with a three-dimensional plot of this profile inserted. To the right of the centre of the membrane we see a region with a negative heat transfer: here, the temperature elevation of the membrane is smaller than that of

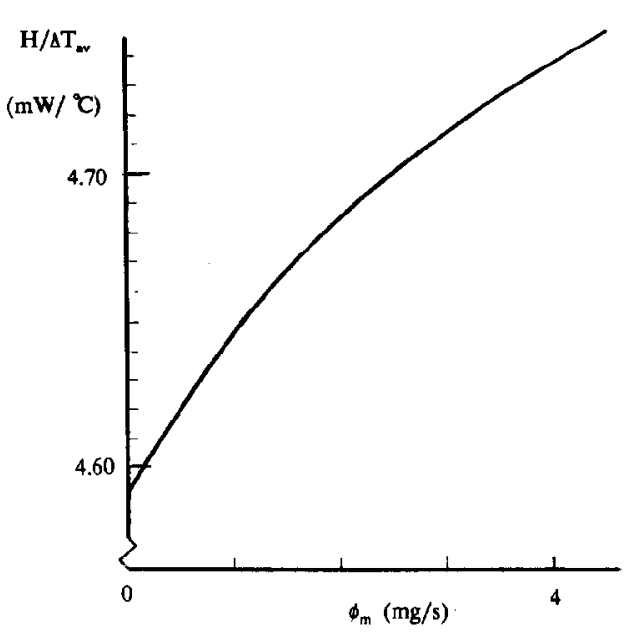

Fig. 7. Total coefficient of heat transfer, $H / \Delta T_{\mathrm{av}}$, as a function of mass flow $\phi_{\mathrm{m}}$, according to the complex model; $4 \times$ $4 \mathrm{~mm} \times 2 \mu \mathrm{m}$ membrane, $800 \times 800 \mu \mathrm{m}$ heat source, $4 \times 4 \mathrm{~mm}$ channel cross-section, $\lambda=157 \mathrm{~W}\left(\mathrm{~m}^{\circ} \mathrm{C}\right)^{-1}, \quad c_{p}=1000 \mathrm{~J}$ $\left(\mathrm{kg}^{\circ} \mathrm{C}\right)^{-1}, \lambda_{\mathrm{g}}=0.027 \mathrm{~W}\left(\mathrm{~m}^{\circ} \mathrm{C}\right)^{-1}[13]$.

the gas, and heat flows back from the gas to the membrane!

This calculation was made for membranes with several thicknesses and lateral dimensions, and for a range of mass flows. A typical result is given in Fig. 7. These calculations show that the shift of the total coefficient of heat transfer is reasonably linear with the mass flow, up to $\phi_{\mathrm{m}}=11\left(\lambda_{\mathrm{g}} / c_{p}\right)\left(l^{2} / h\right)$, and degressing for higher mass flows. This degression is caused by the fact

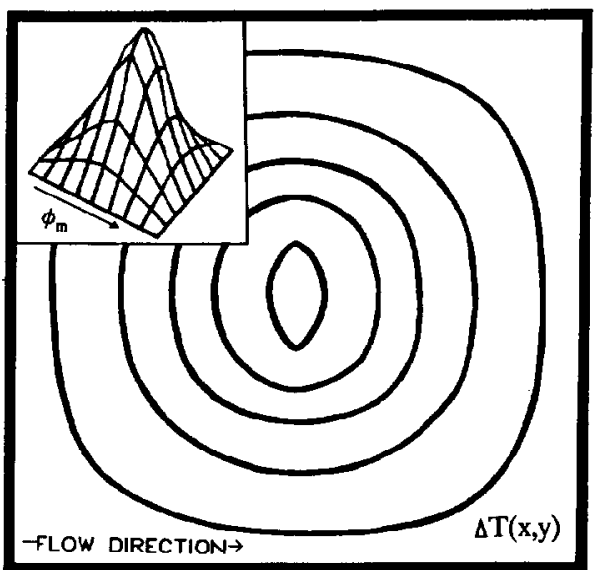

(a)

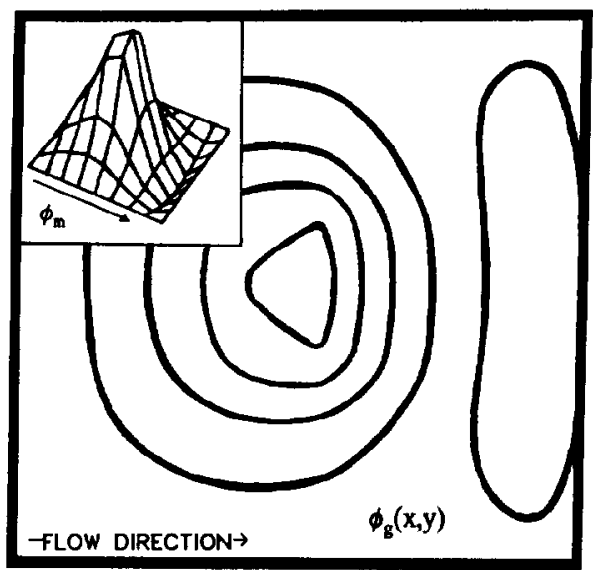

(b)

Fig. 6. Example of a typical result of the complex model for the heat transfer, with a distributed $800 \times 800 \mu \mathrm{m}$ heat source at the centre of a $4 \times 4 \mathrm{~mm}$ membrane. (a) Contour lines and 3-D plot (insert) of the temperature elevation $\Delta T(x, y)$ of the membrane: zero temperature elevation at the edges, maximum values at the centre of the membrane. (b) Contour lines and 3-D plot (insert) of the heat flux $\left(\phi_{\mathrm{g}}(x, y)\right.$ from the membrane to the fluid: zero heat flux at the edges, maximum values at the centre, negative values adjacent to the downstream edge of the membrane. 
that the temperature profile within the gas does not fully develop for high gas velocities, before the gas has passed the heated membrane.

Together with the contribution to the heat transfer by the conduction of heat to the channel walls, we find for the total heat transfer for small mass flows:

$$
\left.\begin{array}{c}
H=\left[14 \lambda t+1.9 \lambda_{\mathrm{g}} \frac{l^{2}}{h}+0.054 c_{p} \phi_{\mathrm{m}}\right] \Delta T_{\mathrm{av}} \\
\text { for } \phi_{\mathrm{m}}<11 \frac{\lambda_{\mathrm{g}}}{c_{\mathrm{p}}} \frac{l^{2}}{h}
\end{array}\right\}
$$

This result shows that the heat transfer by conduction is virtually the same as that predicted by the simple model (1). However, it also shows that the coefficient of heat transfer by convection is approximately ten times smaller than predicted by the simple model, and its relative influence at $10 \mathrm{sccm}$ is only $0.2 \%$ for the membrane with a thickness of $2 \mu \mathrm{m}$. According to the condition for linearity in eqn. (4), the dimensions of the square membrane and of the square cross-section of the flow channel could be scaled down to $l=h=560 \mu \mathrm{m}$, still yielding a linear output in the mass flow range from 0 to $10 \mathrm{sccm}$. Note that in eqn. (4), i.e., the linear part of the curve in Fig. 7 , the additional contribution to the heat transfer by the mass flow is smaller than that by conduction within the gas, with a maximum ratio of $30 \%$.

\section{Resonance Frequency}

We consider the first order of resonance of the membrane, and we neglect the influence of the amplitude of vibration and of damping on the resonance frequency. For an initially flat, square and homogeneous membrane, of uniform thickness and of elastically isotropic material, and clamped at the edges, the dependence of the resonance frequency on the planar stress is approximated by $[14-16]$ :

$$
\begin{aligned}
& f=f_{0}\left[1+0.22 \frac{\sigma_{0}}{\hat{E}}\left(\frac{l}{t}\right)^{2}\right]^{1 / 2} \\
& f_{0}=1.654\left(\frac{\hat{E}}{\rho}\right)^{1 / 2} \frac{t}{l^{2}} \\
& \hat{E}=E /\left(1-v^{2}\right)
\end{aligned}
$$

with $f$ the resonance frequency of the stressed membrane $(\mathrm{Hz}), f_{0}$ the resonance frequency for a stress-free membrane (i.e., a plate) $(\mathrm{Hz}), \sigma_{0}$ the residual stress $\left(\mathrm{N} \mathrm{m}^{-2}\right), E$ Young's modulus $\left(\mathrm{N} \mathrm{m}^{-2}\right), \quad v$ Poisson's ratio, $\rho$ the density $\left(\mathrm{kg} \mathrm{m}^{-3}\right), t$ the thickness of the membrane (m) and $l$ the lateral dimension of the membrane $(\mathrm{m})$.
With a uniform temperature elevation $\Delta T$ of the membrane relative to the substrate, we have a uniform thermal expansion of the membrane $\epsilon_{\mathrm{th}}=\alpha \Delta T$, with $\alpha$ the linear coefficient of expansion. This leads to a compressive planar stress $\sigma_{\mathrm{th}}=(E / 1-v) \epsilon_{\mathrm{th}}$ and a decrease of the resonance frequency. The latter can be found by substituting $\sigma=\sigma_{0}-\left(\frac{E}{1-v}\right) \propto \Delta T$

for the residual stress in eqn. (5).

An initially curved membrane should be regarded as a shell. In that case, the relations (5) and (6) are not adequate. In ref. 17 a model is derived for the dependence of the resonance frequency on a uniform planar stress along the edges, using the Galerkin method. For square shells, simply supported at the edges, we find [18]:

$$
\begin{aligned}
f= & f_{0}\left[1+0.608 \frac{\sigma}{\hat{E}}\left(\frac{l}{t}\right)^{2}+\chi\left[3\left(\frac{w_{1}}{t}\right)^{2}-\left(\frac{w_{0}}{t}\right)^{2}\right]\right]^{1 / 2} \\
\frac{w_{1}}{t} & {\left[1+0.608 \frac{\sigma}{\hat{E}}\left(\frac{l}{t}\right)^{2}+\chi\left[\left(\frac{w_{1}}{t}\right)^{2}-\left(\frac{w_{0}}{t}\right)^{2}\right]\right] } \\
& -\frac{w_{0}}{t}=0
\end{aligned}
$$

$f_{0}=0.907\left(\frac{\hat{E}}{\rho}\right)^{1 / 2} \frac{t}{l^{2}}$

$\chi=0.375\left(1-v^{2}\right)$

with $\sigma$ the uniform stress along the edges $\left(\mathrm{Nm}^{-2}\right)$, $w_{0}$ the height of the shell before stretching (m) and $w_{1}$ the height of the shell after stretching (m) (Fig. 8).

Relation (7) is represented graphically in Fig. 8 for compressive stresses [18]. Due to compressive

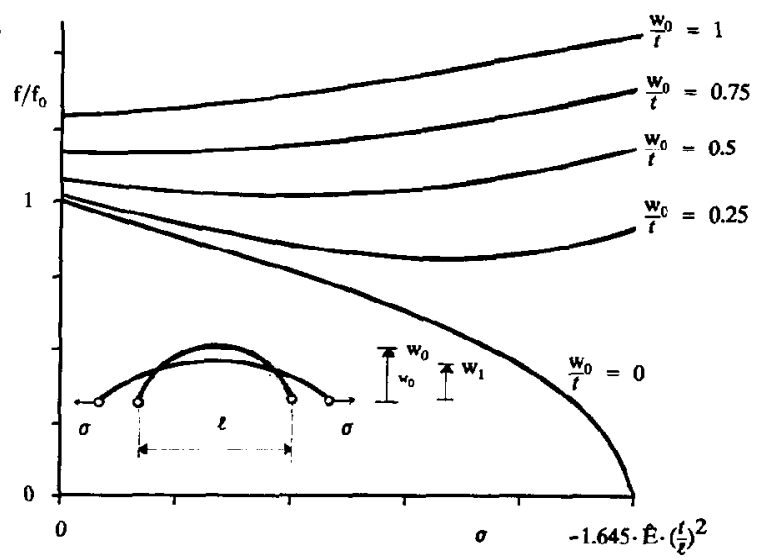

Fig. 8. Calculated resonance frequency $f$ as a function of planar stress $\sigma$ for square homogeneous isotropic shells, simply supported at the edges, according to eqn (7). $w_{0}=$ height of stress-free shell, $w_{1}=$ height of stretched shell. Note: negative values for compressive stress. 
stresses within the shell, the resonance frequency is reduced. Simultaneously, the deflection of the shell is increased. The stiffness of the shell (with respect to bending) increases due to the enlarged curvature; this second effect increases the resonance frequency. From Fig. 8 we see that for small initial deflections and for small compressive stress, the first effect dominates, whereas for larger initial deflections and larger compressive stress, the second effect is larger. Although eqn. (7) was derived for shells with simply supported edges, the results are also qualitatively valid for shells with clamped edges.

\section{Experimental Results}

Figure 9 shows results for the measured resonance frequency for the membrane with a thickness of $2 \mu \mathrm{m}$, together with results for the theoretical average temperature elevation, predicted by the complex model for the heat transfer. Figure 9(a) gives results for the resonance frequency and for the predicted average temperature elevation versus static heat generation, for a zero mass flow. The average temperature elevation is linear with respect to the heat generated on the membrane, with $\chi_{\lambda}=H / \Delta T_{\mathrm{av}}=4.59 \times$ $10^{-3} \mathrm{~W}^{0} \mathrm{C}^{-1}$. Though the predicted temperature changes are small, the resonance frequency changes dramatically, with a maximum differential sensitivity of $1200 \mathrm{~Hz}^{\circ} \mathrm{C}^{-1}$ for $\Delta T_{\mathrm{av}}=8^{\circ} \mathrm{C}$. The dependence of the resonance frequency on the average temperature elevation agrees qualitatively with the predicted behaviour of stressed shells as shown in Fig. 8. Interferographs show that, apart from some bending around the aluminium connection wires, with a deflection of only $25 \mathrm{~nm}$, the membrane is initially flat. However, the membrane
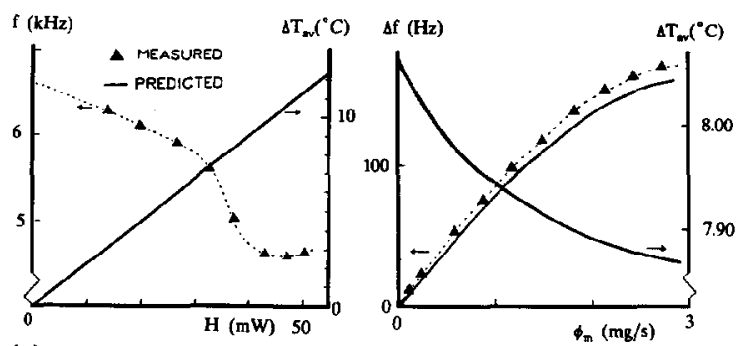

(b)

(a)

Fig. 9. Experimental and theoretical results: (a) measured resonance frequency $f$ and predicted average temperature elevation $\Delta T_{\mathrm{av}}$ as a function of static heat generation $H$ for zero mass flow; (b) measured and predicted resonance frequency shift $\Delta f$ and predicted average temperature elevation $\Delta T_{\mathrm{av}}$ as a function of mass flow $\phi_{\mathrm{m}}$ for $37 \mathrm{~mW}$ static heat generation. bends when its temperature is increased, due to the bending moment caused by the thermal mismatch of the aluminium with the silicon membrane. The deflection increases with increasing power dissipation.

In Fig. 9(b), results are shown for a static heat generation of $37 \mathrm{~mW}$ as a function of the varied nitrogen flow. The predicted average temperature elevation of the membrane and the measured shift of the resonance frequency $\Delta f$ are given. Also, the predicted resonance frequency shift is shown, determined by using the experimental results in Fig. 9(a) for the same values of the predicted average temperature. In the considered mass flow range the predicted and experimental results agree well. The small deviation is probably caused by the uncertainty of the membrane thickness and of the material properties, as well as by omitting the dissipation in the piezoresistors. The dependence of the average temperature elevation of the membrane on the mass flow is small. In the depicted mass flow range, from zero to $3 \mathrm{mg} \mathrm{s}^{-1}$, the predicted average temperature elevation decreases from $8.06{ }^{\circ} \mathrm{C}$ to $7.87^{\circ} \mathrm{C}$. Even so, at a base frequency of $5.0 \mathrm{kHz}$, the measured shift is still $175 \mathrm{~Hz}$ for a mass flow of $3 \mathrm{mg} \mathrm{s}^{-1}$, or $13 \mathrm{~Hz}$ for a mass flow of $10 \mathrm{sccm}$ (i.e., $0.17 \mathrm{mg} \mathrm{s}^{-1}$ ).

We have also carried out these calculations and measurements on samples with membranes $12 \mu \mathrm{m}$ thick [18]. The predicted dependence of the average temperature elevation on the mass flow is six times smaller for these membranes than for the $2 \mu \mathrm{m}$ thick membranes. Also, the predicted influence of the thermal stress is six times smaller. Indeed, for the same value of the predicted average temperature elevation at zero mass flow, the measured resonance frequency shift with increasing mass flow was 36 times less in the considered mass flow range.

\section{Discussion}

The experimental results agree well with the results predicted by the complex model for the heat transfer. Also, the analysis of the resonance frequency is appropriate. The sensitivity of the resonance frequency to a temperature elevation is quite high. This sensitivity can be increased further by fabricating thinner membranes, by applying materials with lower tensile stress and for membranes without initial bending. Materials with a thermal match with the silicon substrate will still be required to prevent stresses building up as a consequence of fluctuations of the ambient temperature. To prevent both the initial bending and the thermal mismatch, metallic connection patterns on top of the micromechanical structure should be avoided. 
For this membrane configuration the sensitivity of the temperature elevation to small mass flows is very small. This sensitivity can be improved by decreasing the heat loss by conduction to the substrate, again by fabricating thinner membranes, or by constructing it from materials with a smaller value for the coefficient of heat conduction than that of single-crystal silicon, e.g., polysilicon [9] and silicon (oxi-)nitride [20].

A further improvement of this sensitivity can be obtained by appling a microbridge rather than a membrane. With only its two shortest edges in thermal contact with the substrate, the heat loss by conduction will be much less for the microbridge than for the membrane. Also, in the case of a microbridge configuration the fluid passes on both sides of the heated structure, whereby, for corresponding conditions, the development of the temperature profile within the gas will be facilitated. Finally, in the flow direction, the temperature profile of the microbridge will decrease much less, and heat will flow from the microbridge to the gas over its entire surface.

Depending on the exact thermal conductivity of the microbridge, the heat transfer by conduction within the gas towards the side walls of the flow channel will be relatively more significant. The complex model for the heat transfer we have described here takes this contribution into account, and therefore can readily be used for the microbridge configuration (provided the width of the bridge is not small compared to the dimensions of the cross section of the flow channel).

The resonance frequency proved to be very stable with respect to small changes in ambient conditions. However, when the sensor is operating at a low temperature elevation, a small change in the temperature of the incoming gas may cause a relatively significant frequency shift. Also, we have seen that the contribution to heat transfer by conduction within the gas is significant. The coefficient of thermal conductivity of gases is largely dependent on temperature, pressure and type of gas [3]. A reference measurement will be required to compensate for such undesired effects [5].

\section{Conclusion}

The possibility of mass flow measurements using a resonant micromechanical structure has been demonstrated. The fabrication of the first prototype consisting of a silicon membrane, with in situ polysilicon thin films for excitation and detection, is relatively simple. The experimental and theoretical results for this resonating membrane prototype show that the numerical model describes the heat transfer very well, and the analysis of the resonance frequency is appropriate.
The sensitivity of the resonance frequency to a temperature elevation is very high. The sensitivity of the temperature elevation to small mass flows, however, can be improved by reducing the heat loss to the substrate by thermal conduction within the micromechanical structure, and by improving the heat transfer from the structure to the gas. This can be achieved by applying alternative configurations and materials.

\section{Acknowledgements}

This project is financed as part of the IOP (Innovative Research Programme) scheme 'Semiconductor Technology' of the Dutch Ministry of Economic Affairs, and by the Department of Applied Physics of the University of Twente. The research is carried out within the Sensors and Actuators Research Unit, and is in cooperation with ASM International/AMTC, Bilthoven, The Netherlands.

The authors would like to thank Mr Gert-Jan Snijders of ASM International/AMTC for the many thoughtful discussions on flow measurement. We would also like to thank Dr Frits van Beckum of the Department of Applied Mathematics for his useful comments on the numerical calculations of the heat transfer. We also thank the technicians of the IC laboratory for allowing us to make use of their LPCVD system. Also, we would like to thank Professor Theo Popma, Professor Jan Fluitman and Dr M. Elwenspoek for their support. Finally, we would like to thank Ms Katrina Emmett for carefully reading the manuscript.

\section{References}

1 Sensor needs in semiconductor plants, J.S.T. News, 3(5) (1984) $31-37$.

2 J. P. DeCarlo, Fundamentals of Flow Measurement, Instrument Society of America, NC, 1983, p. 203.

3 W. M Kays, Convective Heat and Mass Transfer, McGrawHill, New York, 1966, p. 357.

4 J. H. Huijsing, Monolithic flow sensors, a survey, Proc. Solid-State Sensors Conf, Delft, The Netheriands, 1980, pp. $39-52$.

5 R. G. Johnson and R. E. Higashi, A highly sensitive chip microtransducer for air flow and differential pressure sensing applications, Sensors and Actuators, $1 /$ (1987) $63-72$.

6 K. E. Petersen, J. Brown and W. Renken, High-precision, high-performance mass flow sensor with integrated laminar flow micro-channels, Proc. 3rd Int. Conf. Solid-State Sensors and Actuators (Transducers '85), Philadelphia, PA, U.S.A., June 4-11, 1985, pp. 361-363.

7 G. Stemme, An integrated gas flow sensor with pulse-modulated output, Proc. 4th Int. Conf. Solid-State Sensors and Actuators (Transducers '87), Tokyo, Japan, Jume 2-5, 1987, pp. $364-367$

8 M. Esashi, S. Eoh, T. Matsuo and S. Choi, The fabrication of integrated mass flow controllers, Proc. 4h Int. Conf. Solid-State Sensors and Actuators (Transducers '87), Tokyo, Japan, June 2-5, 1987, pp. 830-833. 
9 Y.-C. Tai and R. S. Muller, Lightly-doped polysilicon bridge as a flow meter, Sensors and Actuators, 15 (1988) 63-67.

10 S. Middelhoek, P. J. French, J. H. Huijsing and W. J. Lian, Sensors with digital or frequency output, Sensors and Actuators, 15 (1988) 119-133.

11 T. S. J. Lammerink and W. Wlodarski, Integrated thermally excited resonant diaphragm pressure sensor, Proc. $3 r d$ Int. Conf. Solid-State Sensors and Actuators (Transducers '85), Philadelphia, PA, U.S.A., June 4-11, 1985, pp. 97-100.

12 J. B. Price, Anisotropic etching of silicon with potassium hydroxide-water-isopropyl alcohol, in $\mathbf{H}$. R. Huff and R. R. Burgess (eds.), Semiconductor Silicon 1973, pp. 339353.

13 CRC Handbook of Chemistry and Physics, CRC Press, Boca Raton, FL, U.S.A., 64th edn., 1984.

14 A. W. Leissa, Vibrations of Plates, NASA AP-160, 1969.

15 P. A. A. Laura and E. Romanelli, Vibrations of rectangular plates elastically restrained against rotation along all edges and subjected to a bi-axial state of stress, $J$. Sound Vib., 37 (1974) 367-377.

$16 \mathrm{~S}$. M. Dickinson, The flexural vibration of rectangular orthotropic plates subjected to in-plane forces, J. Appl. Mech., 38 (1971) 699-700.

17 D. Hui and A. W. Leissa, Effects of geometric imperfections on vibrations of biaxially compressed rectangular flat plates, J. Appl. Mech., 50 (1983) 750-756.

$18 \mathrm{G}$. J. Tijhuis, Investigation of the pressure-frequency relationship of a resonating membrane pressure sensor, Master's Thesis (in Dutch), Department of Mechanical Engineering, University of Twente, Enschede, 1987, p. 74.

19 S. Bouwstra, P. Kemna and R. Legtenberg, Thermally excited resonating membrane mass flow sensor, Proc. Eurosensors '87, Cambridge, U.K., Sept. 22-24, 1987, pp. 109-113.

20 S. Bouwstra, R. Legtenberg and Th. J. A. Popma, Silicon rich LPCVD silicon nitride for micro-mechanical structures, Tech. Digest, Eurosensors II, Enschede, The Netherlands, 1988, p. 167.

21 A. R. Mitchell and D. F. Griffiths, Finite Difference Method in Partial Differential Equations, Wiley, New York, 1980.

\section{Biographies}

Siebe Bouwstra was born in Amsterdam, The Netherlands, in 1958. He received his M.Sc. degree in mechanical engineering from the University of Twente, Enschede, The Netherlands, in 1984. In the same year he joined the Sensors and Actuators Research Unit at the University of Twente, where his research is focused on micromechanical resonant sensors and actuators.

Paul Kemna was born in Hengelo, The Netherlands, in 1961. He received his M.Sc. degree in applied physics from the University of Twente, Enschede, The Netherlands, in 1987, after having completed his thesis on the resonating membrane mass flow sensor. $\mathrm{He}$ is presently working for Vredestein B.V., Doetinchem, The Netherlands.

Rob Legtenberg was born in Hengelo, The Netherlands, in 1964. He received his bachelor's degree in applied physics from the Enschede Polytechnic, The Netherlands, in 1985. After having completed his military service, he joined the Sensors and Actuators Research Unit at the University of Twente in 1986.

\section{Appendix: Discretization and Matrix Manipulation}

The two-dimensional Laplace equation for the heat transfer within the membrane (3a), is approximated by differential equations for the temperature elevations at the grid points, using a five-point discretization [21]:

$$
\begin{aligned}
& \lambda t\left\{\left[T_{i+1, j}-2 T_{i, j}+T_{i-1, j}\right] / h_{1}{ }^{2}\right. \\
& \left.\quad+\left[T_{i, j+1}-2 T_{i, j}+T_{i, j-1}\right] / h_{2}{ }^{2}\right\}=-\phi_{i, j}
\end{aligned}
$$

with $T_{i, j}$ the temperature elevation at grid point $i, j$ at $x=i h_{1}, y=j h_{2}, h_{1}$ and $h_{2}$ the distances between the equidistant adjacent grid points in the $x$ - and $y$-directions respectively, and $\phi_{i, j}$ the input of heat in the area $h_{1} h_{2}$ surrounding grid point $i, j$. Note that in (Al) the membrane is assumed to be homogeneous, and of uniform thickness, and the dependence of $\lambda$ on the temperature is omitted.

With the distributed heat generation appropriately attributed to the grid points, and with the boundary condition of zero temperature elevation along the edges, we have a set of $N_{1} N_{2}$ implicit algebraic equations, where $N_{1} N_{2}$ is the number of internal grid points. Writing these in a matrix equation, we have:

$\mathrm{AT}=\phi$

where $\mathrm{T}$ and $\phi$ are $N_{1} N_{2}$ vectors representing the unknown temperature elevations at the sequentially arranged grid points, and the known heat generations in these grid points respectively. $\mathbf{A}$ is the $N_{1} N_{2}$ square matrix with its coefficients determined from the equations (A1):

$\mathbf{A}=\left[\begin{array}{ccccccc}\mathbf{D} & \mathbf{E} & & & & & \\ \mathbf{E} & \mathbf{D} & \mathbf{E} & & & & \\ & \mathbf{E} & \mathbf{D} & \mathbf{E} & & & \\ & & & & \cdot & & \\ & & & & \cdot & & \\ & & & & \mathbf{E} & \mathbf{D} & \mathbf{E} \\ & & & & & \mathbf{E} & \mathbf{D}\end{array}\right]$

with $\mathbf{D}$ and $\mathbf{E}$ submatrices of dimension $\min \left(N_{1}, N_{2}\right)$, if the grid points have been arranged optimally:

$\mathrm{D}=\left[\begin{array}{lllllll}d & e & & & & & \\ e & d & e & & & & \\ & e & d & e & & & \\ & & & \cdot & & & \\ & & & & \cdot & & \\ & & & & e & d & e \\ & & & & & e & d\end{array}\right] \frac{1}{h_{1}^{2}}$ 


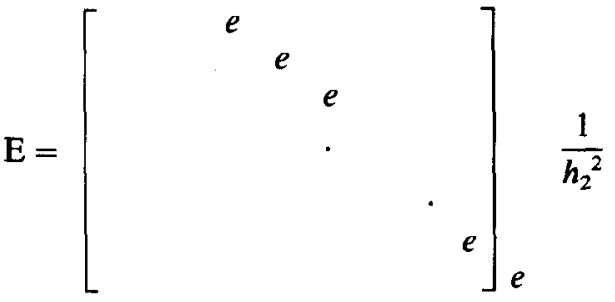

where $e=-1, d=2\left(1+\kappa^{2}\right)$ and $\kappa=h_{1} / h_{2}$.

Note that $A$ is a scarcely filled symmetric band matrix. As only three different values occur for the non-zero coefficients, which are also arranged in a simple pattern, the value of each coefficient is easily determined whenever it occurs in the calculation. Therefore, matrix $A$ does not require large arrays for storage.

Matrix equation (A2) is solved by the Cholesky decomposition technique. In this technique the matrix $\mathbf{A}$ is decomposed in a lower and an upper matrix: $A=L U$, with $L$ a matrix with non-zero coefficients only on its diagonal and below its diagonal, while $U$ a matrix with non-zero coefficients on its diagonal and above. For a band matrix $A$ the matrices $L$ and $U$ are also band matrices, and for a symmetric matrix $A$, the upper matrix $U$ can be chosen to be the transposed matrix of the lower matrix L. Therefore, for computer calculations, $\mathrm{A}$ and its decomposed triangular matrix require only one one-dimensional array of $N_{1} N_{2}\left(\min \left(N_{1}, N_{2}\right)+1\right)$ elements. Using a computer with a fast calculation speed rather than a large storage capacity, even the coefficients of the decomposed matrix can be generated, rather than stored.

For a discretization of ( $3 b)$, describing the heat transfer within the gas, the gas is discretized into parallel planes perpendicular to the flow direction. These planes are discretized into grids, with a five-point discretization of the two-dimensional conduction within these planes similar to that for the membrane. With a backward differentiation of the term in ( $3 b)$ describing the convection, an implicit formulation would result, which is unconditionally stable and convergent with respect to the step size in the $x$-direction. However, the solution of the resulting scheme, similar to that of (A1), leads to an enormous amount of computations.

Alternatively, with a forward differentiation of the convective term, we have:

$$
\begin{aligned}
u_{1, m} & {\left[T_{k+1, l, m}-T_{k, l, m}\right] / h_{3} } \\
& =\frac{\lambda_{g}}{\rho c_{p}}\left\{\left[T_{k, l+1, m}-2 T_{k, l, m}+T_{k, l-1, m}\right] / h_{4}{ }^{2}\right. \\
& \left.+\left[T_{k, l, m+1}-2 T_{k, l, m}+T_{k, l, m-1}\right] / h_{5}{ }^{2}\right\}
\end{aligned}
$$

with $u_{l, m}$ the velocity of the gas in the axial direction at grid points with $y=l h_{4}$ and $z=m h_{5}, T_{k, l, m}$ the temperature elevation at grid point $(x, y, z)=\left(k h_{3}, l h_{4}, m h_{5}\right)$ and $h_{3}, h_{4}$ and $h_{5}$ the grid distances in the $x-, y$ - and $z$-directions respectively.

Note that thanks to the forward differentiation we have explicit expressions for the temperature elevation at each grid point, expressed in the results of the temperature elevations at the grid points of the plane upstream. Therefore, the results for planes further upstream are redundant, and, furthermore, only one array is required for the temperature profiles of the two planes under consideration: partly filled with the results for the lower part of the current plane, and partly filled with the results for the upper part of the former plane. Only the gradients of the temperature profile at the interface with the membrane need to be stored. From these, the components of the heat generation within the membrane are corrected for the next iteration step.

The error in the discretizations (A1) and (A3) are of the order $\left(h_{1}{ }^{2}+h_{2}{ }^{2}\right)$ and $\left(h_{3}+h_{4}{ }^{2}+h_{5}{ }^{2}\right)$ respectively. Obviously, to reach a high accuracy, the grid distances should be chosen as small as possible. As $h_{3}$ has a large influence on the accuracy, the numer of vertical planes for the calculation of the heat transfer within the gas has to be as large as possible. Also, convergence and stability of the calculation based on (A3), requires:

$\frac{\lambda_{\mathrm{g}}}{\rho c_{p}}\left[1 / h_{4}{ }^{2}+1 / h_{5}{ }^{2}\right] \leqslant \frac{1}{2} u_{l, m} / h_{3}$

or, with the velocities $u_{i, m}$ from the profile parabolic with $z$, with its lowest value occurring for $z=h_{5}$, this condition can be rewritten:

$\left[N_{4}{ }^{2}+\left(\frac{l}{h}\right)^{2} N_{5}^{2}\right] \frac{N_{5}}{N_{3}} \leqslant \frac{c_{p} \phi_{m}}{12 \lambda_{g} h}=O[1]$

where $N_{3}, N_{4}$ and $N_{5}$ are the number of grid points in the gas in the $x-, y$-and $z$-directions respectively, $\phi_{\mathrm{m}}$ is the mass flow and $l$ and $h$ are the channel height and width respectively. Again we see that the number of grid points in the $x$-direction should be large, but the demand for convergence and stability imposes limitations on the number of grid points within the vcrtical planes. Conversely, having a set of numbers of grid points $N_{3}, N_{4}$ and $N_{3}$, the calculation has, according to (A4), a lower limit for the mass flow.

For our calculations we made use of the forward differentiation of the convective term, with its explicit formulation. In practice we found that we could go down to mass flows ten times smaller than according to (A4). For smaller mass flows the average temperature elevation of the membrane can then be found from extrapolation. 